\title{
Study on Smart Materials Using in Civil Engineering
}

\author{
Sijia Zhu, ${ }^{1, *}$
}

\author{
${ }^{I}$ School of Architecture and Engineering, North China institute of science and technology, Langfang, Hebei Province, \\ China, 101601 \\ *Corresponding author. Email:1805535214@qq.com
}

\begin{abstract}
Smart materials as the golden key to the development of the 21 st century smart materials have been in many fields to the application and application of ideas. This paper firstly introduces the characteristics of the types of smart materials, and then elaborates on the application scenarios of smart materials through the introduction of common smart materials such as optical fibers, shape memory alloys and piezoelectric materials, and points out the current problems in the field, comparing smart materials and intelligent materials. In conclusion, the future of smart materials is to become more intelligent, which means adding the use of self-controlling. Overcoming all odds, smart materials will play a powerful role in the 21 st century.
\end{abstract}

Keywords: Smart materials, Intelligent material, Civil engineering, Applications \& Performance

\section{INTRODUCTION}

Research into smart materials by engineers and materials scientists has been going on since the 1980s. This is entirely necessary, both in terms of human civilization and the civil engineering profession. The search for more efficient and innovative materials has therefore been a constant goal of generations of researchers.[1]

Since the introduction of smart materials, it has been widely used or conceived for applications in civil engineering. Architecturally, smart materials can bring new inspiration to architectural design and fill in new awareness. Secondly, the use of smart materials contributes to the sustainability of the environment. Structurally, the application of smart materials leads to structural stability and increased safety. The efficiency of the use of the function or form is enhanced. Then it becomes more convenient to carry out maintenance at a later stage, to repair the structure or to use controls that update the original procedures.

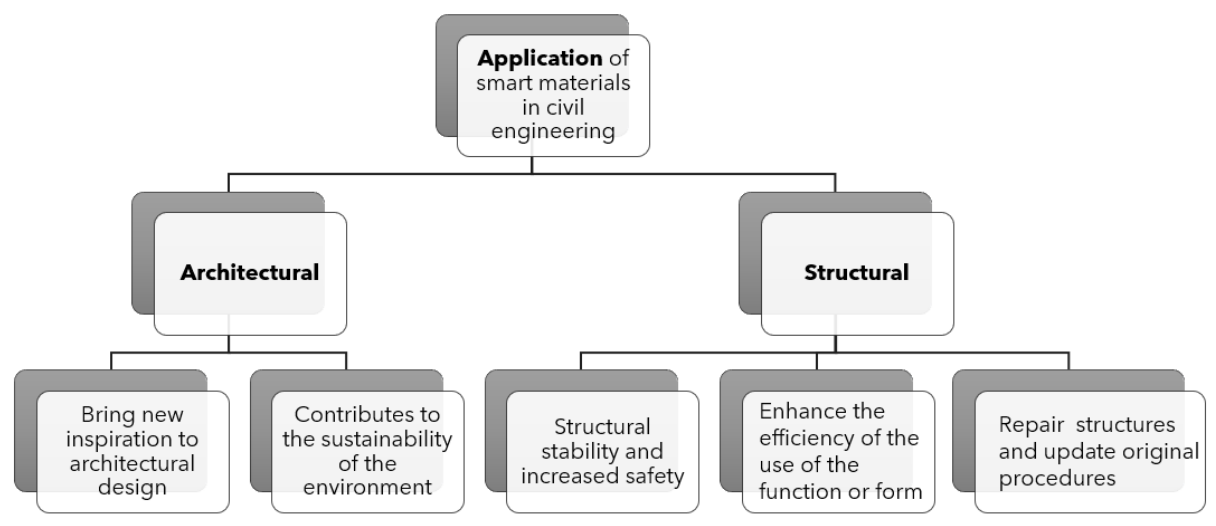

Figure 1 the total application of smart materials in civil engineer 


\section{SMART MATERIALS}

\subsection{Definition of smart materials}

Smart materials exhibit desirable properties when environmental conditions change (Perhaps man-made, perhaps natural). In short, a smart material is one that can respond to any external stimulus to which it is exposed. The stimulus can be electromagnetic waves, pressure, electric currents, ultraviolet light, friction, chemicals, etc. The response can be in the form, size, ductility, color, etc.

\subsection{Types of smart materials}

Smart materials can be broadly divided into two categories according to their functional characteristics. The first is sensing materials, materials that are capable of sensing the intensity of external or internal stimuli such as stress, strain and physio-chemical, light, heat, electricity, magnetism, radiation and other effects, which usually used to make sensors. The other category is actuating materials, which respond to changes in software conditions or internal states. These materials can be made into various actuating or driving elements depending on the environmental requirements or functional requirements.

And in practical engineering applications, the three basic elements we need the materials to have been: sensing, control and actuation. This means that they can sense changes in external stimuli, process information, issue instructions and execute actions, thus realizing a variety of functions such as self-diagnosis, self-monitoring, self-correction and self-adaptation of the structure. As a result, it is difficult to make a single material fulfil all of these functions at the same time, so we usually use a variety of materials to form a new intelligent material structure system and put it into use.

\subsection{Properties of smart materials}

\subsubsection{Bionicity}

Smart materials, which want to give the original traditional materials human characteristics, are above all characterized by their bionic nature. The basic characteristics of its materials are also based on the adaptive systems created by bionics.[2] Human beings will create and improve intelligent materials based on the laws that have been generalized to biological systems.

\subsubsection{Sensorability}

The sensing function is the function of accepting signals from the outside world. In the field of civil engineering, it is possible to establish spatial monitoring of the entire structure. Although smart materials do not have a central system inside them like a conventional CPU, the molecular properties inside them. The recognition of changes in the environment allows this function to be achieved.

\subsubsection{Adaptability}

The most important functional feature we need to introduce smart materials into the civil engineering sector is the ability to adapt. In detail. This means that self-diagnosis and identification can take place during the restoration process. Although the materials do not speak, they can communicate with humans by using special signals that inform the user of their current state.

\subsection{Application of smart materials in civil engineering}

As already mentioned, with the renewal of the construction industry in recent years, smart materials have certain applications in the field of civil engineering structures. At present, intelligent material systems are mainly focused on the following three aspects. One is the real-time detection and monitoring of structural health. The civil engineering sector often has large structures which, once built, are difficult to replace with large component changes. And it is well known that a structure, no matter what material it is made of, will suffer a certain degree of corrosion damage, material ageing and even normal use will lead to some reduction in the load-bearing capacity of the structure, even if it is in a normal external environment for a long time.

This is why we need an efficient and convenient structural health inspection system for timber projects, in order to determine in real time whether defects exist, where they are located and the extent of damage, so that the structure can be repaired or reinforced in time to prevent irreparable damage and ensure the integrity and safety of the structure.

Secondly, shape adaptive materials are combined with traditional building materials. Adaptive structures can withstand both load transfer forces and at the same time have the ability to detect physical indicators of the structure (stress, strain, temperature, cracks, etc.) and to change some of the structural properties (inherent frequency, electromagnetic field distribution, etc.) The third is the use of smart materials for structural dynamic response. The third is the use of smart materials for structural dynamic response problems. For example, in large structures such as high-rise buildings or bridges, where seismic and wind control is important, the incorporation of intelligent material structural systems can provide a more effective solution to the problem and enable the initial display of adaptive control of the structure. 


\section{SOME TYPICAL SMART MATERIALS USING IN CIVIL ENGINEERING}

\subsection{Piezoelectric materials}

Piezoelectricity, which is said to be the most effective mechanism for making mainly sensors and actuators. Piezoelectric materials can be divided into single-phase piezoelectric materials, piezoelectric composites and inorganic piezoelectric materials. The main principle of operation is the piezoelectric effect. By applying a force to the material to produce a deformation, an equal amount of dissimilar charge is generated at the two end electrodes and the charge density is proportional to the magnitude of the force, and vice versa for the inverse piezoelectric effect. Therefore, using the positive piezoelectric effect, piezoelectric materials are made into sensing elements that can monitor changes in charge, i.e., sense the vibration modes of a structure. On the basis of the above, control and quantification of the piezoelectric input using suitable algorithms can be achieved for structural vibration. However, piezoelectric materials are costly and are often used in electronic software and not in traditional construction materials, but in comparison his use is even more remarkable.

\subsection{Shape memory alloys}

Shape memory alloys have a shape memory function. Shape change can stimulate this property, while generating high reversion stresses and reversion strains that can store and transmit high strength energy. Materials based on this property are embedded in various structures and used as intelligent actuators to enhance the adaptive, self-diagnostic and self-enhancing capabilities of structures for the study of cracks, damage, deformation and vibration.[3]

The pseudo-elasticity of the phase change and the hysteresis of the phase change allow the stress-strain curve to take on a circular shape during loading or unloading, allowing large amounts of energy to be consumed or absorbed. They are often used as passive energy dissipators or passive energy dissipation control systems. They are usually installed between layers or at the base of a structure in order to sense the interlayer deformation more precisely and to consume seismic energy.

\subsection{Optical fibers}

Optical fibers have the ability to transmit information like no other material. For this reason, they are used as the sensing material of choice. The optical fiber is embedded in a structure and when the structure is deformed or cracked due to stress or temperature changes, the optical fiber is deformed, resulting in a change in the optical signal (e.g., light intensity, phase, wavelength, etc.), which allows information on the stress, strain, temperature and cracks in the material surrounding the fiber to be obtained for real-time online inspection of the structure.

Optical fiber sensing mechanisms are divided into two categories: direct measurement and interferometric measurement. Direct measurement is the direct measurement of the change in light intensity within an optical fiber caused by mechanical changes. Interferometric methods measure the amount of change in optical range and phase to obtain mechanical parameters within the concrete, also known as optical interferometry.

It is also worth mentioning that the optical fibers are in a two-layer tubular structure. The inner layer is a very ductile silica and the outer layer is a transparent protective layer. The cost of making optical fibers is therefore not prohibitive and they are widely used for the detection of temperatures within concrete structures.

\subsection{Carbon fiber reinforced concrete (CFRC)}

Carbon fiber itself is a high strength, high modulus material with good electrical conductivity. Adding the right amount of carbon fiber to concrete can give it intrinsic self-sensing properties and drive function. The strength and toughness of the concrete is significantly improved. [4]

In addition, carbon fibers and carbon fibers between the cement particles, hydration products and other combinations to form a conductive network with a certain resistance. This varies with stress and can richly reflect the damage within the structure. For example, a definite change in resistivity represents an elastic change in the material and an irreversible change in resistivity represents inelastic deformation and fracture. It is also temperature sensitive. The potential energy generated by the temperature difference makes the structure more sensitive.

There are two types of carbon fiber. The way the material is joined, short cut chaotically distributed, and continuous carbon fiber bundles reinforced singly. The latter gives the full tensile strength of the carbon fibers, but is too expensive to build. So, the two methods compared to the former is more widely used. 
Table 1 the comparison of four classic smart materials

\begin{tabular}{|c|c|c|c|c|}
\hline & $\begin{array}{c}\text { Piezoelectic } \\
\text { materials }\end{array}$ & $\begin{array}{c}\text { Shape memory } \\
\text { alloys }\end{array}$ & Optical fiber & CFRC \\
\hline Advantages & $\begin{array}{l}\text { High stability, } \\
\text { high sensitivity }\end{array}$ & $\begin{array}{c}\text { Elastic in nature, } \\
\text { high strength }\end{array}$ & $\begin{array}{l}\text { the ability to } \\
\text { transmit } \\
\text { information }\end{array}$ & $\begin{array}{l}\text { High strength } \\
\text { light weight }\end{array}$ \\
\hline Disadvantages & $\begin{array}{l}\text { not used in } \\
\text { traditional } \\
\text { construction } \\
\text { materials }\end{array}$ & $\begin{array}{l}\text { Catch rust quickly } \\
\text { environmental } \\
\text { pollution }\end{array}$ & - & $\begin{array}{l}\text { Costly. } \\
\text { No compressive } \\
\text { strength. } \\
\text { non-recyclable }\end{array}$ \\
\hline Cost & +++ & + & ++ & +++++ \\
\hline Application & Roads, buildings & $\begin{array}{c}\text { Earthquake } \\
\text { proof houses } \\
\text { bridges }\end{array}$ & $\begin{array}{l}\text { Detect the } \\
\text { temperatures within } \\
\text { concrete structures }\end{array}$ & $\begin{array}{c}\text { Some delicate } \\
\text { design } \\
\text { sensitive work } \\
\text { high-end projects }\end{array}$ \\
\hline
\end{tabular}

\section{CONSTRAINTS TO THE DEVELOPMENT OF SMART MATERIALS}

When any new material is first introduced it is always met with skepticism. It is human nature to be afraid of trying new things, especially when existing building materials can still be used. But if smart materials are widely used in public buildings, or if they are supported by the state, this idea will change.[5]

At present, smart materials are still an emerging field with little research, resulting in practitioners not yet having an accurate knowledge and grasp of these materials. In particular, for example, the aforementioned piezoelectric materials that are often used in electronic components. Because the development of electronic elements is slow, its value cannot be fully reflected, which implicates various fields and requires interlocking progress.

One of the most limiting points in the development of smart materials is the high cost he brings, while the material is often used in large structures extremely costly.[6] But later, as demand increases, mass production will bring costs down. The efficiency of using smart materials will also increase considerably.

In addition, smart materials use the principles of bionics but have never actually got the essence of it and today are only able to detect damage in small repair actions and cannot prevent predictive warning of large disasters. This means that the material cannot reach a truly high level of performance.

\section{FUTURE WORKS}

When talking about the future of smart materials, there is one concept needs to be understood in advance. That is "intelligent materials", which can summarize from some past scientific literature or common sense that: the ideal intelligent material should have the ability to perceive external stimuli, learn for itself and respond optimally.[7] By means of different external stimuli, better responses are made. Why is this idea being put forward? What is the difference between these two similar concepts? As already mentioned, smart materials have three primary functions. In fact, in summary, there are only two, sensing and responding. And the intelligence of intelligent materials can be referred to artificial intelligence, human intelligence and other related terms. From this, it can be seen that intelligent materials add new functions of self-optimization and improvement to the foundation of smart materials. In my opinion, this is the future goal for the development of smart materials. Figuratively speaking, if you think of material as a human brain, the thinking from stimulus to response is no longer monolithic and linear. The material itself can do kinds of optimized thinking and make choices that are better suited to the performance of the application.

We expect scientists in the future to tackle this area. Thinking in terms of atoms or molecules, fusing different materials together or tapping into new properties of known materials, let the applications that smart materials can bring to the civil engineering field are even more amazing.

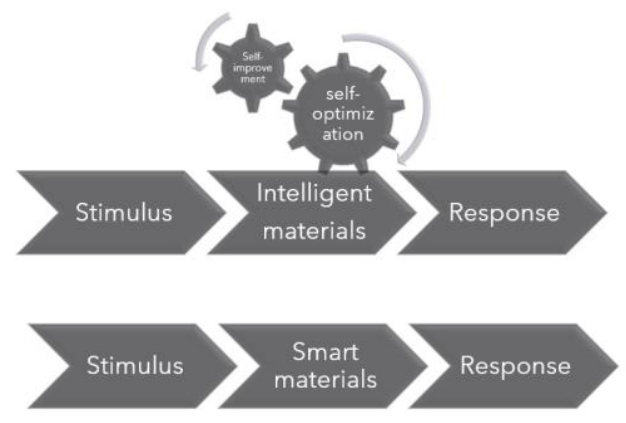

Figure 2 intelligent materials VS smart materials

\section{CONCLUSION}

In the near future, intelligent material systems will demonstrate amazing capabilities and facilitate further 
breakthroughs in engineering. Figuratively speaking, the use of intelligent materials is equivalent to adding a brain capable of thinking to traditional buildings, when a bridge has a problem with concrete somewhere the brain makes an autonomous judgement to repair it; an eye capable of observing, if a deformation or crack exists somewhere in the aircraft, advance warning is given; an arm capable of waving, when a large change in the external conditions of the structure occurs, the "muscles in the arm " move autonomously to counteract noise or vibration. Smart materials will be the key to unlocking developments in the 21 st century, so let's look forward to more of his wonders for the civil engineering sector.

\section{REFERENCES}

[1] Cao Zhaoping, Wang Sheliang. Application of intelligent material structure system in civil engineering $[\mathrm{J}]$. Journal of Chongqing University of Architecture, 2001(01):108-113.

[2] Wang Dongfeng. The development and application of intelligent materials in civil engineering [J]. Construction Technology Development,2021,48(02):62-64.

[3] Iman Abavisani, Omid Rezaifar, Ali Kheyroddin,Multifunctional properties of shape memory materials in civil engineering applications: A state-of-the-art review,Journal of Building Engineering,Volume 44,2021,102657

[4] Zhang Weidong, Xu Xueyan. Application of intelligent materials in health monitoring of civil engineering $[\mathrm{J}]$. Petroleum Engineering Construction,2004(02):9-14+63.

[5] Abeer Samy Yousef Mohamed,Smart Materials Innovative Technologies in architecture; Towards Innovative design paradigm,Energy Procedia,Volume 115,2017,Pages 139-154,

[6] Abhilash Mukherjee, Deepmala, Prateek Srivastava, Jasminder Kaur Sandhu,Application of smart materials in civil engineering: A review, Materials Today: Proceedings,2021,

[7] Kai Liu, Mike Tebyetekerwa, Dongxiao Ji, Seeram Ramakrishna,Intelligent Materials,Matter,Volume 3, Issue 3,2020,Pages 590-593, 